\title{
Photospheric high-frequency acoustic power excess in sunspot umbra: signature of magneto-acoustic modes
}

\author{
S. Zharkov ${ }^{1}$, S. Shelyag ${ }^{2}$, V. Fedun ${ }^{3,4}$, R. Erdélyi ${ }^{4}$, and M. J. Thompson ${ }^{4,5}$ \\ ${ }^{1}$ Department of Physics and Mathematics, University of Hull, Cottingham Road, Kingston-upon-Hull, HU6 7RX, UK \\ ${ }^{2}$ Monash Centre for Astrophysics, School of Mathematical Sciences, Monash University, Clayton, Victoria, 3800, Australia \\ ${ }^{3}$ Dept of Automatic Control and Systems Engineering, The University of Sheffield, Mappin Street, Sheffield, S1 3JD, UK \\ ${ }^{4} \mathrm{SP}^{2} \mathrm{RC}$, School of Mathematics and Statistics, Univ. of Sheffield, Hounsfield Road, Hicks Building, Sheffield, S3 7RH, UK \\ ${ }^{5}$ High Altitude Observatory, P.O. Box 3000, Boulder, CO 80307-3000, USA
}

Correspondence to: S. Zharkov (s.zharov@ hull.ac.uk)

Received: 24 March 2013 - Revised: 13 June 2013 - Accepted: 28 June 2013 - Published: 6 August 2013

\begin{abstract}
We present observational evidence for the presence of MHD (magnetohydrodynamic) waves in the solar photosphere deduced from SOHO/MDI (Solar and Heliospheric Observatory/Michelson Doppler Imager) Dopplergram velocity observations. The magneto-acoustic perturbations are observed as acoustic power enhancement in the sunspot umbra at high-frequency bands in the velocity component perpendicular to the magnetic field. We use numerical modelling of wave propagation through localised nonuniform magnetic field concentration along with the same filtering procedure as applied to the observations to identify the observed waves. Guided by the results of the numerical simulations we classify the observed oscillations as magnetoacoustic waves excited by the trapped sub-photospheric acoustic waves. We consider the potential application of the presented method as a diagnostic tool for magnetohelioseismology.
\end{abstract}

Keywords. Solar physics, astrophysics, and astronomy (magnetic fields; photosphere and chromosphere)

\section{Introduction}

Helioseismology, the study of acoustic oscillations excited by turbulence in the convection zone of the Sun, has been hugely successful in developing and testing our theories and models of solar interior (Duvall et al., 1997). Investigations of sunspots using methods of local helioseismology (Kosovichev and Duvall, 1997; Zhao and Kosovichev, 2006; Zharkov et al., 2007; Thompson and Zharkov, 2008; Gizon et al., 2009), which analyse the properties of waves passing through these magnetic features, have provided us with a wealth of insight into their subphotospheric nature and, in many cases, have posed new questions concerning the understanding of sunspot structure and its interaction with waves.

So far such studies have mostly concentrated on the effect of sunspot magnetic structure on acoustic oscillations present in the quiet Sun photosphere. At the same time, it is known from MHD (magnetohydrodynamic) theory that a number of various oscillatory modes are present in magnetised atmospheres (Pintér and Erdélyi, 2011). In fact, it is argued (Moradi and Cally, 2008; Moradi et al., 2009) that, at least some of, the inconsistencies in the helioseismic analyses of sunspots (Gizon et al., 2009) are likely due to not taking these modes into account. Numerical MHD simulations are currently used to help us gain an insight into such problems (Crouch and Cally, 2003; Shelyag et al., 2007, 2009, 2010; Parchevsky and Kosovichev, 2007; Cameron et al., 2008; Khomenko et al., 2009; Felipe et al., 2010). A variety of propagating and standing MHD waves (e.g. slow mode, Alfvén, and fast mode) have been observed higher in the outer atmosphere of the Sun, mainly in coronal loops, but also in other structures such as coronal plumes and prominences (Bogdan et al., 2003; Nagashima et al., 2007; Fedun et al., 2009; Jess et al., 2009; Zaqarashvili and Erdélyi, 2009; Morton et al., 2011, 2012). In addition, numerous observations obtained in various spectral lines revealed presence of three minute umbral oscillations from the transition region into the corona (e.g. Centeno et al., 2006; Banerjee et al., 2007; Sych et al., 2012). However, limited observational 

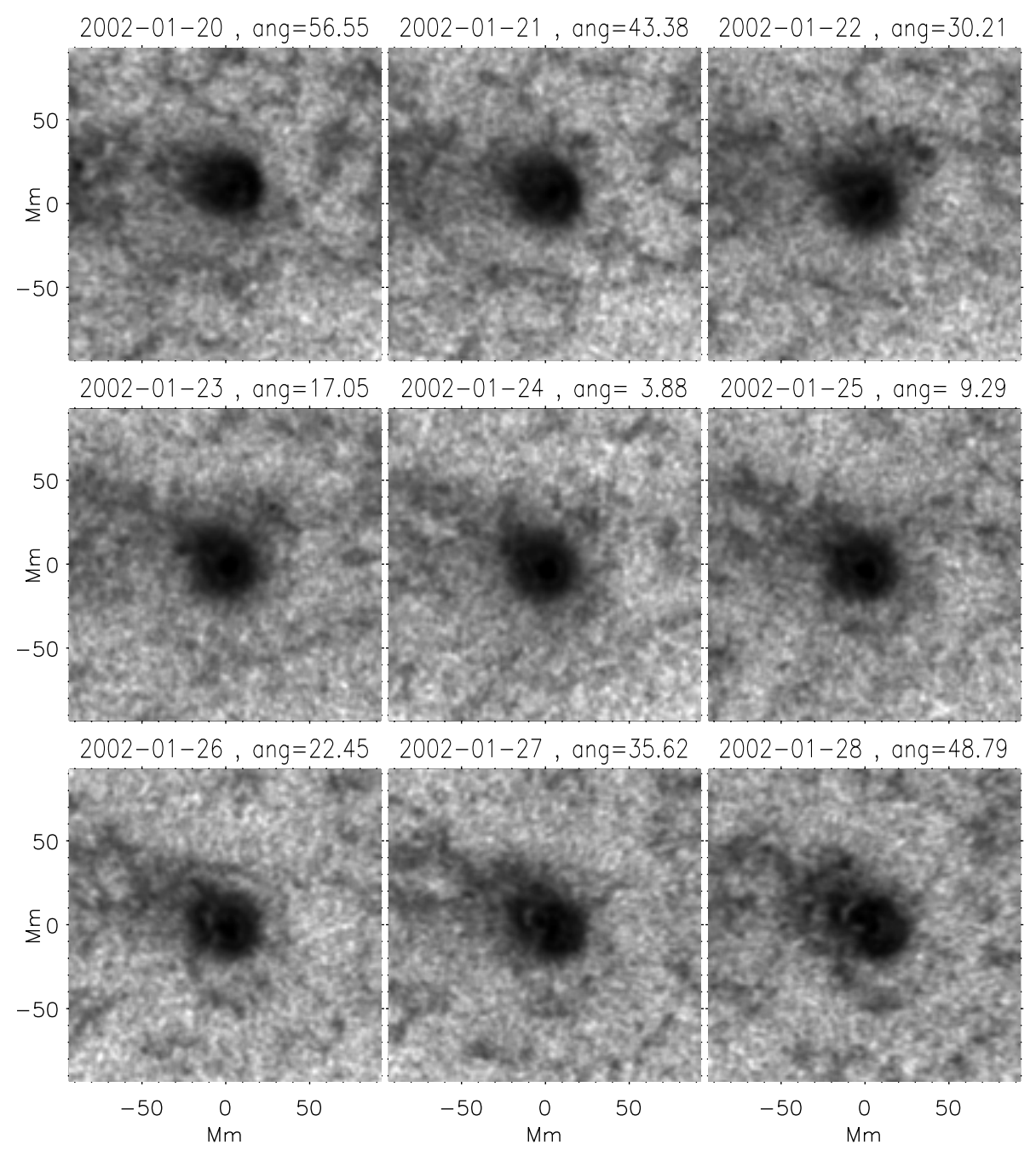

Fig. 1. Acoustic power estimated from the line-of-sight velocity data for the NOAA AR9787 region. The data are taken at 9 subsequent snapshots as the sunspot travels from $56^{\circ}$ West to $61^{\circ}$ East on the solar surface. The data are frequency-filtered with the filter frequency band centred on $3 \mathrm{mHz}$. The images show little apparent variation in the centre of the sunspot.

evidence of magneto-acoustic waves has been found so far at the level of the solar photosphere (Zirin and Stein, 1972; Dorotovič et al., 2008; Morton et al., 2011, 2013).

Based on preliminary analysis of symbiosis of observational and simulated data, in this paper we present a first direct evidence of presence of magneto-acoustic waves in the sunspot umbra. Comparing the results of MHD forward modelling with the observations we show that the umbral power increase at high frequencies seen at large angles between the normal to the solar surface and the line of sight is consistent with the slow high- $\beta$ and fast low- $\beta$ waves.

\section{Data and reduction}

We investigate data from NOAA Active Region 9787, consisting of a nearly single axisymmetric sunspot that showed little evolution during 20-28 January 2002, and observed continuously by the SOHO Michelson Doppler Imager (MDI) instrument. MDI uses the spectral line $\mathrm{Ni}$ I $6776.772 \AA$ originating at approximately $300 \mathrm{~km}$ height above the solar surface (Scherrer et al., 1995). The dataset, available on the European Helio- and Asteroseismology Network (HELAS) website at http://www.mps.mpg.de/ projects/seismo/NA4/DATA/data_access.html, was prepared by HELAS and has been thoroughly described and analysed by Gizon et al. (2009). The images were remapped using Postel projection with a map scale of $0.12^{\circ}$ to one $512 \times 512 \times 1440$ data cube of Doppler velocity data for each day. The centres of projection were chosen to track the motion of the sunspot (Carrington longitude of $\approx 133^{\circ}$ and latitude at $8.3^{\circ} \mathrm{S}$ ). Over the nine days of observations the region travelled from $56^{\circ} \mathrm{W}$ to $61^{\circ} \mathrm{E}$.

For each day of observation we compute the temporal Fourier transform of the Doppler images. We then divide this 


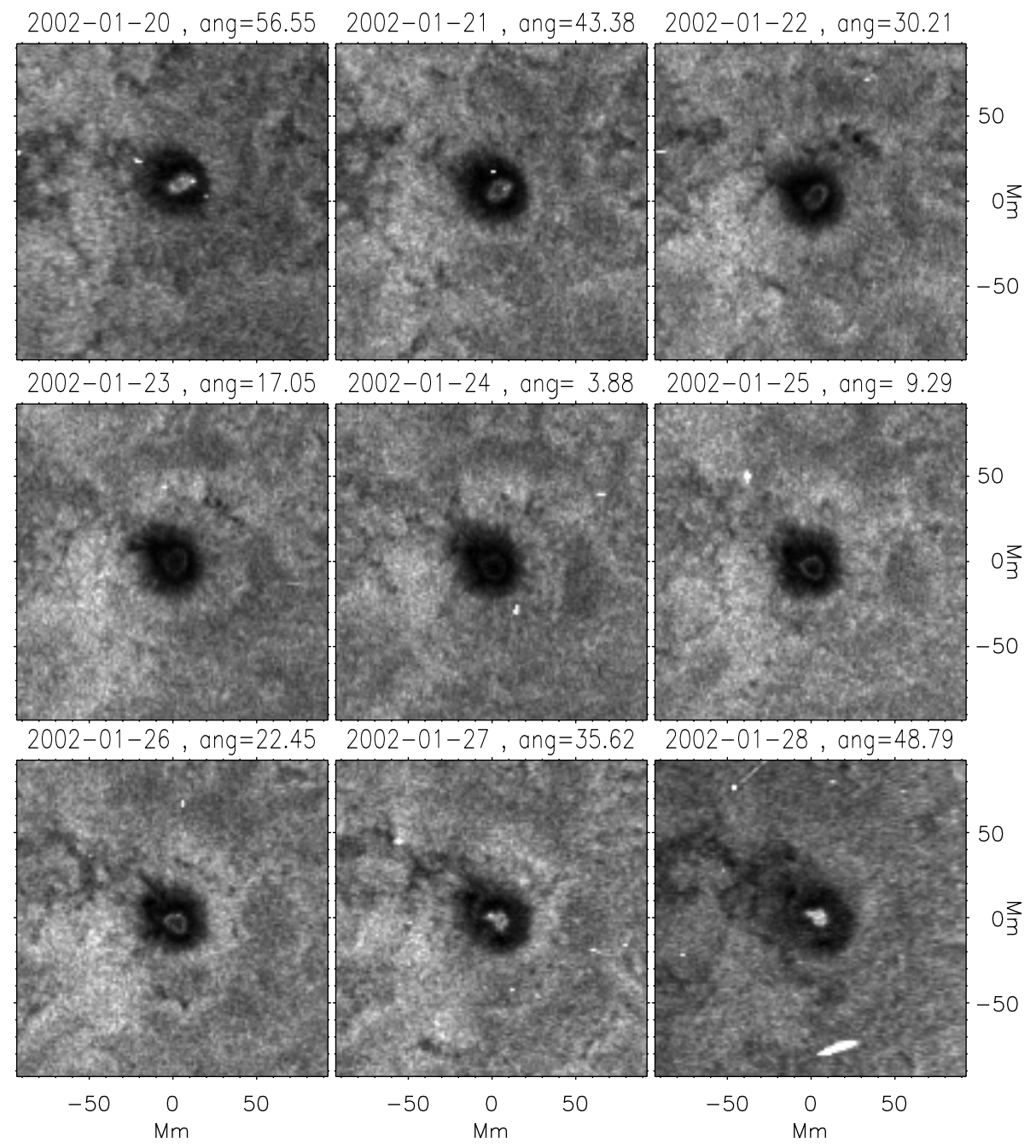

Fig. 2. As Fig. 1 but for the frequency band centred $6 \mathrm{mHz}$. The images show a significant variation dependent on the heliographic angle and reveal easily noticeable power enhancement inside the sunspot at large angles from the solar disk centre (first and last frames of the panel).

into $1 \mathrm{mHz}$ bandwidth intervals and estimate the oscillatory power averaged over each of these frequency bandwidths. The power is then normalised by the dominating quiet Sun acoustic power value. Figures 1 and 2 show the results for the 3 and $6 \mathrm{mHz}$ centred frequency bands, respectively.

\section{Simulation}

We applied the code SAC (Sheffield Advanced Code) to carry out the simulations of sound wave propagation through a localised strong non-uniform magnetic field concentration, representing a sunspot. A detailed description of the code, numerical methods and the tests to show the robustness and applicability of SAC to a wide variety of magnetohydrodynamic problems are presented by Shelyag et al. (2008).

The code solves the full compressible system of MHD equations in three, $(x, y, z)$ with $z$ axis in the vertical di- rection, or two, $(x, z)$ with all variables independent of $y$, dimensions on Cartesian grid. Hyperdiffusivity and hyperresistivity techniques are used to ensure the numerical solution is stable (Nordlund and Galsgaard, 1995). The code also uses variable separation to conserve the magneto-hydrostatic equilibrium of the background unperturbed state.

Standard Model S (Christensen-Dalsgaard et al., 1996), slightly modified to achieve convective stability, is implemented as the unperturbed "quiet", non-magnetic model of the solar interior. The physical size of the computational domain is $180 \mathrm{Mm}$ in the horizontal and $50 \mathrm{Mm}$ in the vertical directions, respectively. The domain is resolved by $960 \times 1000$ grid cells. The numerical domain is set such that the upper boundary is located right above the visible solar surface.

The boundaries of the domain are open, allowing the plasma to move into and out of the numerical domain freely. However, some weak reflection of the waves from the 

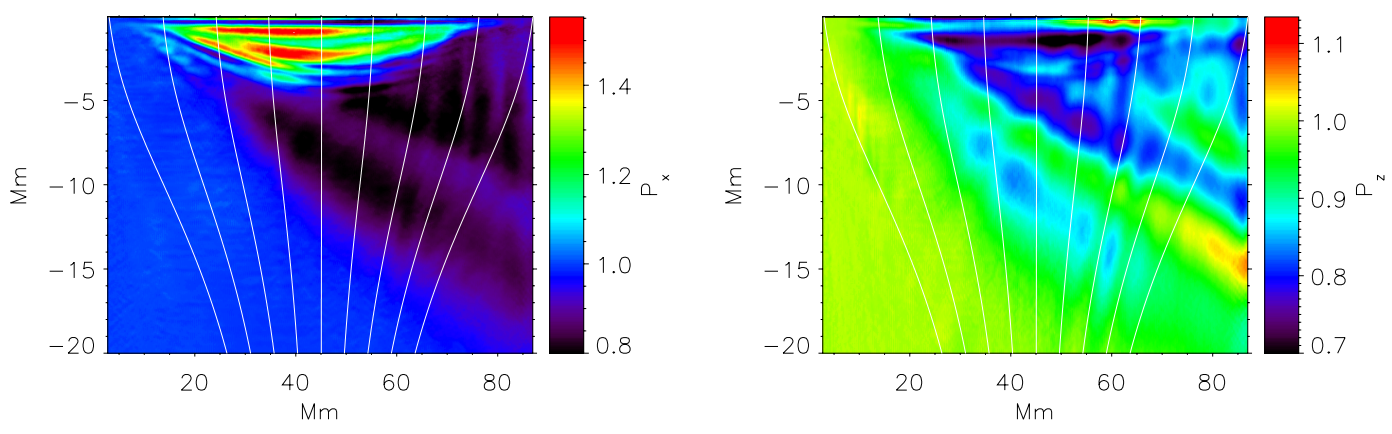

Fig. 3. Horizontal (left panel) and vertical (right panel) acoustic power ratios between the quiet and magnetic parts of the synthetic data obtained by means of forward modelling. The magnetic field lines are overplotted. Strong absorption is observed in the vertical acoustic power in the magnetised region, while the horizontal power component shows a strong enhancement. Note that the scaling is different in vertical and horizontal directions is different, thus the magnetic field lines in the plots do not show the actual inclination of the magnetic field in the model.

boundaries is observed due to the not-ideal numerical representation of the boundary conditions.

A non-uniform non-potential self-similar static magnetic field configuration (see Schlüter and Temesváry, 1958; Deinzer, 1965; Schüssler and Rempel, 2005; Shelyag et al., 2010, 2009; Fedun et al., 2011a,b,c) is implemented in half of the domain to mimic sunspot properties. The maximum vertical magnetic field strength is $3.5 \mathrm{kG}$ at the level approximately corresponding to the visible solar surface. The magnetic field of this strength not only decreases the temperature in the sunspot at the solar surface, but also creates a layer with the ratio of local Alfvén speed to local sound speed greater than unity. This layer may be responsible for magneto-acoustic wave mode conversion.

We use a single spatially and temporally localised acoustic source to excite the oscillations in the domain. The source is located in the middle of the horizontal layer $500 \mathrm{~km}$ beneath the solar surface. Due to symmetry of the source in the $x$ direction, this allows us to directly compare the character of the wave propagation in the magnetic and non-magnetic halves of the domain.

\section{Results and discussion}

The simulation used here was analysed using time-distance helioseismology, outlined in Shelyag et al. (2009) as case B where additional details about the magnetic configuration can be found. The source generates acoustic wave-packet propagating through magnetic and non-magnetic halves of the simulation domain. When travelling through magnetised plasma in 2-D, the acoustic wave generally splits into slow and fast magneto-acoustic waves. However, in the high- $\beta$ region covering most of the computational domain except layers near the top, kinetic pressure dominates over magnetic. Thus, as shown in Shelyag et al. (2009), the fast high- $\beta$ wave generally dominates until it reaches the mode-conversion region, $v_{\mathrm{A}} \approx v_{\mathrm{s}}$ i.e. $\beta \approx 1$, of the magnetic flux tube situ- ated near the top of the domain. Then another mode becomes prominent in the magnetised domain, clearly seen in the computed magnetic field perturbation movies which can be found at http://robertus.staff.shef.ac.uk/publications/ acoustic/. Therefore the mode is magnetic in nature.

By considering the velocities in non-magnetic and magnetic parts of the same simulation we have observed that this mode is more pronounced in the horizontal rather than vertical component. This can be clearly seen in Fig. 3, where we have measured the acoustic power in the simulation box separately for horizontal and vertical velocity components, $P_{x \mid z}(x, z)=\int v_{x \mid z}^{2}(x, z, t) d t$ and then constructed the acoustic power ratios between "quiet" and "magnetic" parts of the simulation as function of horizontal coordinate and depth. The acoustic power measured in the horizontal component of the velocity shows a pronounced enhancement in the near surface layers.

From theory, the wave group speed is related to the energy propagation. For the high- $\beta$ plasma slow-mode wave it equals to the Alfvén speed and is almost parallel to the magnetic field. For the high- $\beta$ fast the wave group speed is nearly independent of the magnetic field (Priest, 1987; Syrovatskii and Zhugzhda, 1967). To investigate these perturbations further we consider vertical propagation of the waves at the centre of the flux tube, $x_{\mathrm{c}}$, by cross-correlating the near surface velocity component measured at $z=z_{\text {surf }}$ with deeper signal $C(z, t)=\int v_{x \mid z}\left(x_{\mathrm{c}}, z_{\text {surf }}, \tau\right) v_{x \mid z}\left(x_{\mathrm{c}}, z, t+\tau\right) d \tau$. At the centre of the flux tube the magnetic field is exactly vertical by construction and the magnetic field line is parallel to the $z$ axis. The results of cross-correlation are presented in Fig. 4 for the horizontal and vertical velocity components. For both components, we see the wave packet arriving to the surface from negative time lag, being then reflected back to the interior for positive times. However, the correlation in the horizontal velocity component also shows the second, slower, mode propagating along the $z$ direction with the local Alfvén speed. Thus, we conclude that, this is the slow-mode high- $\beta$ wave 

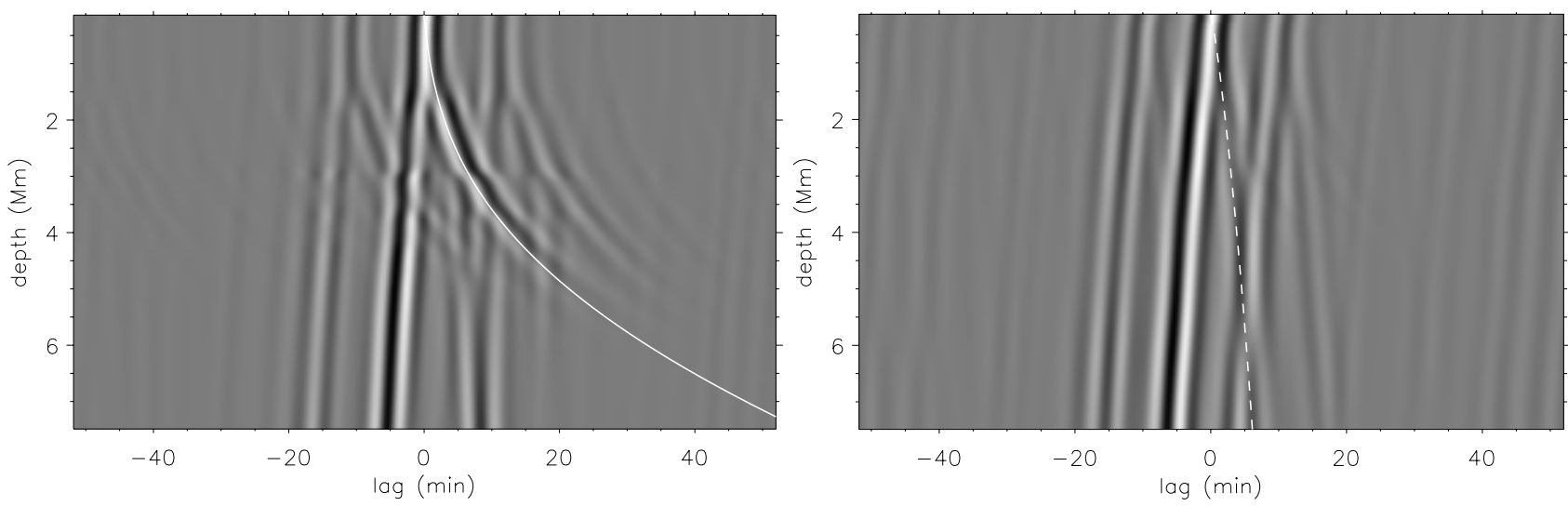

Fig. 4. Simulation cross-correlation in $v_{x}$ (left panel) and $v_{z}$ (right panel) at the centre of the magnetic flux tube as a function of depth. The arriving fast magneto-acoustic wave and reflected slow wave can be seen in the $v_{x}$ cross-correlation, however, the fast wave dominates the $v_{z}$ cross-correlation image. The theoretical time-distance curve for the wave propagating with Alfvén velocity is overplotted on the left panel (solid white line), and time-distance curve for the wave propagating with local sound speed is overplotted on the right one (dashed line).

that propagates along the magnetic field lines generated by the mode conversion of the original wave. Note that while this mode travels along the field line, directed in $z$ direction in this case, it is virtually absent in velocity $z$ component (right plot in Fig. 4), which is in agreement with the theory (e.g. Priest, 1987). The counterpart of this mode above the modeconversion region $(\beta<1)$ is the fast low- $\beta$ mode, which also travels at Alfvén speed and oscillates in the direction normal to it (Priest, 1987; Khomenko and Cally, 2011, 2012).

Thus we conclude that for almost vertical magnetic field, the slow high- $\beta$ mode will mainly contribute to the horizontal component of velocity.

In our 2-D model we can describe the line-of-sight velocity $v_{\mathrm{LOS}}=v_{z} \cos \alpha+v_{x} \sin \alpha$, where $\alpha$ is the angle between the normal to the surface and line-of-sight direction. Clearly as $\alpha$ approaches $\pi / 2$ the horizontal components dominate

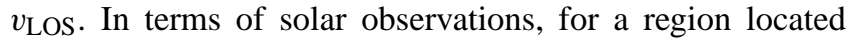
near the equator, $\alpha$ is approximately equal to the heliographic longitude of the observed region, therefore we can expect the stronger contribution of such magnetic modes at larger heliographic angles. The data in Figs. 1 and 2 show the acoustic power ratios in frequency bands centred at 3 and $6 \mathrm{mHz}$ measured from daily NOAA 9787 data from 21 January $\left(\alpha \approx 56^{\circ} \mathrm{W}\right)$, when the region was located close to the west limb, to 24 January $\left(\alpha \approx 3^{\circ} \mathrm{W}\right)$, when it was at the central meridian, to 28 January (close to east limb, $\alpha \approx 61^{\circ} \mathrm{E}$ ). At $6 \mathrm{mHz}$ we can see the power enhancement (brightening) at the central part of the sunspot, for the observations made close to the limb. No such effect is observed at $3 \mathrm{mHz}$. Also, ring-like structures of increased acoustic power are visible at the sunspot centre at high frequencies at smaller heliographic angles, which suggests a possible signature of strong inclined fields.

Such observational frequency dependence is also supported by our numerical simulation. Figure 5 shows that the horizontal component oscillations inside the flux tube are strongly suppressed at $3 \mathrm{mHz}$, and enhanced at $6 \mathrm{mHz}$ and above. Also in the vertical component the power increase at high frequencies is observed in the region where the magnetic field becomes slightly inclined. This could correspond to aforementioned ring-like structures seen at high frequencies in observations close to the central meridian.

In order to verify our results, we consider the possible artifacts that might be present in the near limb data and can potentially affect the results of the analysis. SOHO/MDI are known to suffer from low light level "saturation" problem as well as from limitations of on-board processing algorithm. We have carefully considered the following potential artefacts: pixel rotation, low light levels and limitations of onboard processing algorithm (Liu and Norton, 2001). Firstly, the pixel rotation does not cause a problem at the limb where the slow mode measurements are carried out. Also, following Scherrer (1993) we have implemented the on-board algorithm, and using the intensity data from continuum observations, we have run several test simulations with varying noise levels. The results have shown little or no ill effect for acoustic power computation. Thus, we conclude that the acoustic power measurements derived from the SOHO/MDI instrument are not affected by such issues.

\section{Conclusions}

We have found observational evidence for magneto-acoustic waves deduced from high frequency acoustic power maps constructed from near-the-limb observations. Near-the-limb the observed Doppler shifted line-of-sight velocity signal has a strong horizontal, i.e. perpendicular to magnetic field, component. This behaviour agrees with theoretical description of the slow high- $\beta$ and fast low- $\beta$ modes. Although torsional Alfvén-type motions can be present in sunspots, here we 

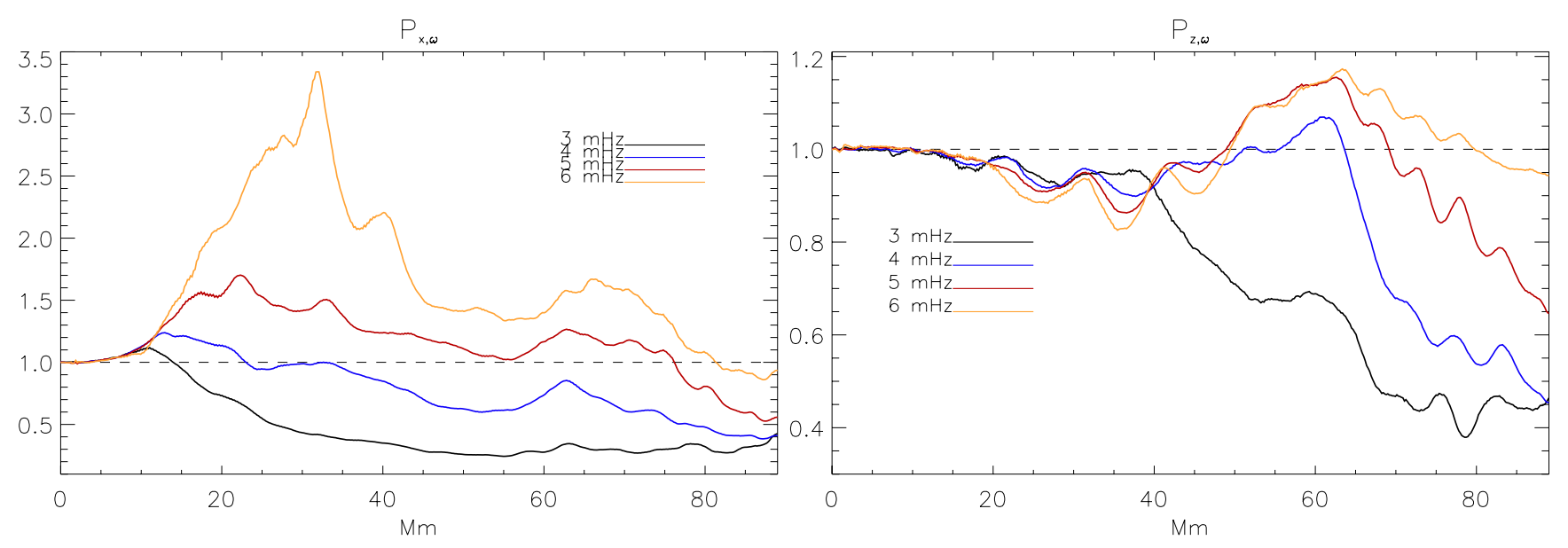

Fig. 5. The acoustic power ratio in horizontal (left plot) and vertical (right plot) velocity components in the simulation domain, filtered using the same filters as applied to observational data and described in Sect. 2. A strong power enhancement at $6 \mathrm{mHz}$ in the horizontal velocity component is observed, which corresponds to the power enhancement seen in the $6 \mathrm{mHz}$ filtered observations (see Fig. 2). Again, no power enhancement is observed in the $3 \mathrm{mHz}$ frequency band (cf. Fig. 1).

were able to explain the excess power with two-dimensional numerical model where Alfvén waves are prohibited to propagate.

Supported by our simulation, we propose that in the high$\beta$ interior most of the acoustic wave packet energy, when passing through the flux tube, goes into the fast-mode high$\beta$ wave. This wave when travelling through the conversion region $v_{\mathrm{A}} \approx v_{\mathrm{s}}$, splits into fast and slow magneto-acoustic waves in our simulation. The fast magneto-acoustic high- $\beta$ wave passing through the tube interior transforms back to the acoustic mode, while the slow high- $\beta$ mode travels down along the field lines.

The fact that such slow oscillations are better pronounced in the direction normal to the magnetic field, opens a number of interesting questions and possibilities for helioseismic analysis of sunspot properties. This approach allows us to investigate the dependency between magnetic field angle at the surface, line of sight and oscillatory power, thus potentially providing information about magnetic field inclination. For example, our findings can be related to acoustic halo results reported in Schunker and Braun (2011), where strong correlation of the enhanced high-frequency power with magneticfield inclination was reported. It could also be of interest to model the effects of the acoustic source depth on geometry and interaction with magnetic field (Zharkov, 2013; Cally, 2013).

Finally, in this work we have used a HELAS dataset of over nine days of continuous observations of an isolated large and stable sunspot located at relatively low latitude. It is the subject of future research to develop this analysis further and to extend it to other more extensive observations, for instance, using SDO (Solar Dynamics Observatory) data building on acoustic power analysis carried out in Rajaguru et al. (2012).
Acknowledgements. This work was initiated when all of the authors worked at School of Mathematics and Statistics, University of Sheffield. We would like to thank HELAS and for providing the data and support. We also thank Tom Duvall, Phil Scherrer and Michael Ruderman for helpful discussions and guidance. R. Erdélyi acknowledges M. Kéray for patient encouragement and is also grateful to NSF, Hungary (OTKA, Ref. Nos. K67746, K83133) for support received. S. Shelyag's research is supported by the Australian Research Council Future Fellowship. The authors would also like to thank the referees for their comments and input which have improved the paper.

Guest Editor M. Gedalin thanks M. Ruderman and one anonymous referee for their help in evaluating this paper.

\section{References}

Banerjee, D., Erdélyi, R., Oliver, R., and O'Shea, E.: Present and Future Observing Trends in Atmospheric Magnetoseismology, Sol. Phys., 246, 3-29, 2007.

Bogdan, T. J., Carlsson, M., Hansteen, V. H., McMurry, A., Rosenthal, C. S., Johnson, M., Petty-Powell, S., Zita, E. J., Stein, R. F., McIntosh, S. W., and Nordlund, Å.: Waves in the Magnetized Solar Atmosphere. II. Waves from Localized Sources in Magnetic Flux Concentrations, Astrophys. J., 599, 626-660, doi:10.1086/378512, 2003.

Cally, P. S.: Near- and Far-field Response to Compact Acoustic Sources in Stratified Convection Zones, Astrophys. J., 768, 35, doi:10.1088/0004-637X/768/1/35, 2013.

Cameron, R., Gizon, L., and Duvall Jr., T. L.: Helioseismology of Sunspots: Confronting Observations with Three-Dimensional MHD Simulations of Wave Propagation, Sol. Phys., 251, 291308, doi:10.1007/s11207-008-9148-1, 2008.

Centeno, R., Collados, M., and Trujillo Bueno, J.: Spectropolarimetric Investigation of the Propagation of Magnetoacoustic Waves and Shock Formation in Sunspot Atmospheres, Astrophys. J., 640, 1153-1162, doi:10.1086/500185, 2006. 
Christensen-Dalsgaard, J., Dappen, W., Ajukov, S. V., Anderson, E. R., Antia, H. M., Basu, S., Baturin, V. A., Berthomieu, G., Chaboyer, B., Chitre, S. M., Cox, A. N., Demarque, P., Donatowicz, J., Dziembowski, W. A., Gabriel, M., Gough, D. O., Guenther, D. B., Guzik, J. A., Harvey, J. W., Hill, F., Houdek, G., Iglesias, C. A., Kosovichev, A. G., Leibacher, J. W., Morel, P., Proffitt, C. R., Provost, J., Reiter, J., Rhodes Jr., E. J., Rogers, F. J., Roxburgh, I. W., Thompson, M. J., and Ulrich, R. K.: The Current State of Solar Modeling, Science, 272, 1286-1292, doi:10.1126/science.272.5266.1286, 1996.

Crouch, A. D. and Cally, P. S.: Mode Conversion of Solar $p$ Modes in non-Vertical Magnetic Fields - i. two-Dimensional Model, Sol. Phys., 214, 201-226, doi:10.1023/A:1024247617255, 2003.

Deinzer, W.: On the Magneto-Hydrostatic Theory of Sunspots, Astrophys. J., 141, 548, doi:10.1086/148144, 1965.

Dorotovič, I., Erdélyi, R., and Karlovský, V.: Identification of linear slow sausage waves in magnetic pores, in: Proceedings of the International Astronomical Union, IAU Symposium, volume 247, Waves \& Oscillations in the Solar Atmosphere: Heating and Magneto-Seismology, Porlamar, Isla de Margarita, Venezuela, 17-22 September 2007, p. 351-354, 2008.

Duvall Jr., T. L., Kosovichev, A. G., Scherrer, P. H., Bogart, R. S., Bush, R. I., de Forest, C., Hoeksema, J. T., Schou, J., Saba, J. L. R., Tarbell, T. D., Title, A. M., Wolfson, C. J., and Milford, P. N.: Time-Distance Helioseismology with the MDI Instrument: Initial Results, Sol. Phys., 170, 63-73, doi:10.1023/A:1004907220393, 1997.

Fedun, V., Erdélyi, R., and Shelyag, S.: Oscillatory Response of the 3D Solar Atmosphere to the Leakage of Photospheric Motion, Sol. Phys., 258, 219-241, doi:10.1007/s11207-009-9407-9, 2009.

Fedun, V., Shelyag, S., and Erdélyi, R.: Numerical Modeling of Footpoint-driven Magneto-acoustic Wave Propagation in a Localized Solar Flux Tube, Astrophys. J., 727, 17, doi:10.1088/0004-637X/727/1/17, 2011a.

Fedun, V., Shelyag, S., Verth, G., Mathioudakis, M., and Erdélyi, R.: MHD waves generated by high-frequency photospheric vortex motions, Ann. Geophys., 29, 1029-1035, doi:10.5194/angeo29-1029-2011, 2011b.

Fedun, V., Verth, G., Jess, D. B., and Erdélyi, R: Frequency Filtering of Torsional Alfvén Waves by Chromospheric Magnetic Field, Astrophys. J. Lett., 740, L46, doi:10.1088/20418205/740/2/L46, 2011c.

Felipe, T., Khomenko, E., and Collados, M.: Magneto-acoustic Waves in Sunspots: First Results From a New Three-dimensional Nonlinear Magnetohydrodynamic Code, Astrophys. J., 719, 357-377, doi:10.1088/0004-637X/719/1/357, 2010.

Gizon, L., Schunker, H., Baldner, C. S., Basu, S., Birch, A. C., Bogart, R. S., Braun, D. C., Cameron, R., Duvall, T. L., Hanasoge, S. M., Jackiewicz, J., Roth, M., Stahn, T., Thompson, M. J., and Zharkov, S.: Helioseismology of Sunspots: A Case Study of NOAA Region 9787, Space Sci. Rev., 144, 249-273, doi:10.1007/s11214-008-9466-5, 2009.

Jess, D. B., Mathioudakis, M., Erdélyi, R., Crockett, P. J., Keenan, F. P., and Christian, D. J.: Alfvén Waves in the Lower Solar Atmosphere, Science, 323, 1582-1585, doi:10.1126/science.1168680, 2009.

Khomenko, E. and Cally, P. S.: Numerical simulations of conversion to Alfvén waves in solar active regions, in: Journal of Physics
Conference Series 271/GONG-SoHO 24: A new era of seismology of the sun and solar-like stars, 27 June-2 July 2010, Aix-enProvence, France, 012042, 2011.

Khomenko, E. and Cally, P. S.: Numerical Simulations of Conversion to Alfvén Waves in Sunspots, Astrophys. J., 746, 68, doi:10.1088/0004-637X/746/1/68, 2012.

Khomenko, E., Kosovichev, A., Collados, M., Parchevsky, K., and Olshevsky, V.: Theoretical Modeling of Propagation of Magnetoacoustic Waves in Magnetic Regions Below Sunspots, Astrophys. J., 694, 411-424, doi:10.1088/0004-637X/694/1/411, 2009.

Kosovichev, A. G. and Duvall Jr., T. L.: Acoustic tomography of solar convective flows and structures, in: Astrophysics and Space Science Library, Vol. 225/SCORe'96: Solar Convection and Oscillations and their Relationship, Aarhus, Denmark, 27-31 May 1996, p. 241-260, 1997.

Liu, Y. and Norton, A. A.: MDI Measurement Errors: The Magnetic Perspective, SOI Technical Note 01-144, http://soi.stanford.edu/ technotes/01.144/TN01-144.pdf, 2001.

Moradi, H. and Cally, P. S.: Time - Distance Modelling in a Simulated Sunspot Atmosphere, Sol. Phys., 251, 309-327, doi:10.1007/s11207-008-9190-z, 2008.

Moradi, H., Hanasoge, S. M., and Cally, P. S.: Numerical Models of Travel-Time Inhomogeneities in Sunspots, Astrophys. J. Lett., 690, L72-L75, doi:10.1088/0004-637X/690/1/L72, 2009.

Morton, R. J., Erdélyi, R., Jess, D. B., and Mathioudakis, M.: Observations of Sausage Modes in Magnetic Pores, Astrophys. J. Lett., 729, L18, doi:10.1088/2041-8205/729/2/L18, 2011.

Morton, R. J., Verth, G., Jess, D. B., Kuridze, D., Ruderman, M. S., Mathioudakis, M., and Erdélyi, R.: Observations of ubiquitous compressive waves in the Sun's chromosphere, Nature Communications, 3, 1315, doi:10.1038/ncomms2324, 2012.

Morton, R. J., Verth, G., Fedun, V., Shelyag, S., and Erdélyi, R.: Evidence for the Photospheric Excitation of Incompressible Chromospheric Waves, Astrophys. J., 768, 17, doi:10.1088/0004637X/768/1/17, 2013.

Nagashima, K., Sekii, T., Kosovichev, A. G., Shibahashi, H., Tsuneta, S., Ichimoto, K., Katsukawa, Y., Lites, B., Nagata, S., Shimizu, T., Shine, R. A., Suematsu, Y., Tarbell, T. D., and Title, A. M.: Observations of Sunspot Oscillations in G Band and CaII H Line with Solar Optical Telescope on Hinode, Pub. Astron. Soc. Japan, 59, 631-636, 2007.

Nordlund, Å. and Galsgaard, K.: Technical Report, Copenhagen: Astronomical Observatory, 1995.

Parchevsky, K. V. and Kosovichev, A. G.: Three-dimensional Numerical Simulations of the Acoustic Wave Field in the Upper Convection Zone of the Sun, Astrophys. J., 666, 547-558, doi:10.1086/520108, 2007.

Pintér, B. and Erdélyi, R.: Effects of Magnetic Fields in the Solar Atmosphere on Global Oscillations, Space Sci. Rev., 158, 471504, doi:10.1007/s11214-011-9805-9, 2011.

Priest, E. R.: Solar magneto-hydrodynamics, edited by: Priest, E. R., Dordrecht: D. Reidel, 1987.

Rajaguru, S. P., Couvidat, S., Sun, X., Hayashi, K., and Schunker, H.: Properties of High-Frequency Wave Power Halos Around Active Regions: An Analysis of Multi-height Data from HMI and AIA Onboard SDO, Sol. Phys., 303, published online first, doi:10.1007/s11207-012-0180-9, 2012. 
Scherrer, P. H.: MDI IP Velocity Algorithm, SOI Technical Note 94-110, http://soi.stanford.edu/technotes/94.110.pdf, 1993.

Scherrer, P. H., Bogart, R. S., Bush, R. I., Hoeksema, J. T., Kosovichev, A. G., Schou, J., Rosenberg, W., Springer, L., Tarbell, T. D., Title, A., Wolfson, C. J., Zayer, I., and MDI Engineering Team: The Solar Oscillations Investigation - Michelson Doppler Imager, Sol. Phys., 162, 129-188, doi:10.1007/BF00733429, 1995.

Schlüter, A. and Temesváry, S.: The Internal Constitution of Sunspots, in: Proceedings from IAU Symposium no. 6/Electromagnetic Phenomena in Cosmical Physics, Stockholm, Sweden, 27-31 August 1956, p. 263-274, 1958.

Schunker, H. and Braun, D. C.: Newly Identified Properties of Surface Acoustic Power, Sol. Phys., 268, 349-362, doi:10.1007/s11207-010-9550-3, 2011.

Schüssler, M. and Rempel, M.: The dynamical disconnection of sunspots from their magnetic roots, Astron. Astrophys., 441, 337-346, doi:10.1051/0004-6361:20052962, 2005.

Shelyag, S., Erdélyi, R., and Thompson, M. J.: Forward modelling of sub-photospheric flows for time-distance helioseismology, Astron. Astrophys., 469, 1101-1107, doi:10.1051/00046361:20066946, 2007

Shelyag, S., Fedun, V., and Erdélyi, R.: Magnetohydrodynamic code for gravitationally-stratified media, Astron. Astrophys., 486, 655-662, doi:10.1051/0004-6361:200809800, 2008.

Shelyag, S., Zharkov, S., Fedun, V., Erdélyi, R., and Thompson, M. J.: Acoustic wave propagation in the solar sub-photosphere with localised magnetic field concentration: effect of magnetic tension, Astron. Astrophys., 501, 735-743, doi:10.1051/00046361/200911709, 2009.
Shelyag, S., Mathioudakis, M., Keenan, F. P., and Jess, D. B.: A photospheric bright point model, Astron. Astrophys., 515, A107, doi:10.1051/0004-6361/200913846, 2010.

Sych, R., Zaqarashvili, T. V., Nakariakov, V. M., Anfinogentov, S. A., Shibasaki, K., and Yan, Y.: Frequency drifts of 3-min oscillations in microwave and EUV emission above sunspots, Astron. Astrophys., 539, A23, doi:10.1051/0004-6361/201118271, 2012.

Syrovatskii, S. I. and Zhugzhda, Y. D.: Oscillatory Convection of a Conducting Gas in a Strong Magnetic Field, Astron. Zh+., 44, $1180,1967$.

Thompson, M. J. and Zharkov, S.: Recent Developments in Local Helioseismology, Sol. Phys., 251, 225-240, doi:10.1007/s11207008-9143-6, 2008.

Zaqarashvili, T. V. and Erdélyi, R.: Oscillations and Waves in Solar Spicules, Space Sci. Rev., 149, 355-388, doi:10.1007/s11214009-9549-y, 2009.

Zhao, J. and Kosovichev, A. G.: Surface Magnetism Effects in Time-Distance Helioseismology, Astrophys. J., 643, 1317-1324, doi:10.1086/503248, 2006.

Zharkov, S.: Geometric properties of acoustic waves generated by a point source in the solar-like interior: effects of acoustic cutoff frequency, Mon. Not. R. Astron. Soc., 431, 3414-3428, doi:10.1093/mnras/stt420, 2013.

Zharkov, S., Nicholas, C. J., and Thompson, M. J.: Time distance study of isolated sunspots, Astron. Nachr., 328, 240-244, doi:10.1002/asna.200610744, 2007.

Zirin, H. and Stein, A.: Observations of Running Penumbral Waves, Astrophys. J. Lett., 178, L85-L87, doi:10.1086/181089, 1972. 